\title{
Directrices para el diseño de aplicaciones usables en entornos de televisión digital interactiva'
}

\section{Guidelines for Designing Usable Applications in Interactive Digital Television Environments ${ }^{2}$}

\author{
Andrés Solano ${ }^{3}$ \\ Gabriel Chanchí \\ César Collazos ${ }^{5}$ \\ José Luis Arciniegas ${ }^{6}$ \\ Cristian Rusu ${ }^{7}$
}

D01:10.11144/JaverIana.IYU18-1.DDAU

\begin{abstract}
'Fecha de recepción: 30 de enero de 2013. Fecha de aceptación: 26 de junio de 2013. Este trabajo ha sido parcialmente financiado por el proyecto UsabiliTV: framework para la evaluación de la usabilidad de aplicaciones en entornos de televisión digital interactiva, código 1103521 28462, financiado por Colciencias. Además, ha sido financiado por el Programa Nacional para Estudios de Doctorado en Colombia año 2011, de Colciencias. Este trabajo ha sido desarrollado por el grupo de Investigación y Desarrollo en Ingeniería de Software (IDIS) de la Universidad del Cauca, Colombia, y el grupo de investigación en Interacción Persona-Computador (UseCV) de la Pontificia Universidad Católica de Valparaíso, Chile.

${ }^{2}$ Reception date: January 30th, 2013. Acceptance date: June 26th, 2013. This work has been partially supported by the project UsabiliTV: Framework para la evaluación de la usabilidad de aplicaciones en entornos de televisión digital interactiva, ID 1103521 28462, funded by Colciencias. and the Programa Nacional para Estudios de Doctorado en Colombia año 2011, funded by Colciencias. This work was highly supported by the IDIS Research Group of University of Cauca, Colombia, and by the UseCV Research Group in Human - Computer Interaction of the Pontifical Catholic University of Valparaiso, Chile. ${ }^{3}$ Magíster en Computación, Universidad del Cauca, Popayán, Colombia. Estudiante de Doctorado en Ciencias de la Electrónica, Universidad del Cauca. Correo electrónico: afsolano@unicauca.edu.co.

${ }^{4}$ Ingeniero en Electrónica y Telecomunicaciones, Universidad del Cauca, Popayán, Colombia. Estudiante de Doctorado en Telemática de la Universidad del Cauca. Correo electrónico: gabrielc@unicauca.edu.co.

${ }^{5}$ Doctor en Ciencias Mención Computación, Universidad de Chile, Santiago, Chile. Profesor titular del Departamento de Sistemas, Universidad del Cauca, Popayán, Colombia. Correo electrónico: ccollazo@unicauca.edu.co.

${ }^{6}$ Doctor en Ingeniería de Sistemas Telemáticos, Universidad Politécnica de Madrid, España. Profesor titular del Departamento de Telemática, Universidad del Cauca, Popayán, Colombia. Correo electrónico: jlarci@unicauca.edu.co

${ }^{7}$ Doctor en Ciencias de la Ingeniería, Universidad Técnica de Cluj-Napoca, Rumania. Profesor titular de la Escuela de Ingeniería Informática, Pontificia Universidad Católica de Valparaíso, Chile. Correo electrónico: cristian.rusu@ucv.cl.
\end{abstract}




\section{Resumen}

Con el inminente despliegue que tendrá la televisión digital en los próximos años, tras el apagón analógico y considerando que la interactividad es una de sus principales ventajas, se requiere un estudio de la usabilidad de las aplicaciones interactivas en las cuales el usuario participa de manera activa durante la emisión de un contenido multimedia. Por lo anterior, es necesario encontrar el modo más adecuado para diseñar aplicaciones usables en entornos de televisión digital interactiva (TDi). Este artículo presenta un conjunto de directrices para el diseño de aplicaciones usables en entornos de TDi, propuestas a partir de un conjunto de evaluaciones de usabilidad realizadas en el Laboratorio de Televisión Digital de la Universidad del Cauca de Colombia.

\section{Palabras clave}

Televisión digital interactiva, usabilidad, directrices, aplicaciones, interactividad.

\section{Keywords plus}

Digital televisión, digital media.

\section{Abstract}

Digital television will have an imminent deployment in the next coming years, and considering that interactivity is one of the main advantages of this kind of systems, it is required a study of the usability of interactive applications in which the user actively participates during the transmission of a television program. Therefore it is necessary to find the best way to design usable applications in interactive Digital Television environments (iTV). This paper presents a set of guidelines supporting design of usable applications in iTV environments, which have been proposed from a set of usability evaluations conducted at the Digital Television Laboratory of the Universidad del Cauca (Colombia).

\section{Keywords}

Interactive digital television, usability, guidelines, applications, interactivity.

\section{Palabras clave descriptores}

Televisión digital, formatos digitales. 


\section{Introducción}

En la actualidad se tiene la posibilidad de contar con diversos medios de comunicación (internet, dispositivos móviles inteligentes, entre otros), caracterizados por un notable incremento en la interactividad con el usuario, así como por la posibilidad de personalizar la presentación de la información y la sencillez al utilizarlos (Collazos et al., 2009). A pesar de la aparición de estos nuevos medios de comunicación, la televisión tradicional no ha perdido su vigencia y sigue siendo uno de los medios de comunicación más utilizados en el mundo (Collazos et al., 2008). Simultáneamente, los usuarios y sus necesidades crecen y cambian rápidamente, a medida que avanza la tecnología, por lo que es necesario lograr una apropiación adecuada por parte de los usuarios que utilicen la televisión digital interactiva (TDi), de tal manera que estén cómodos y puedan suplir sus necesidades con su uso.

La principal ventaja que ofrece la TDi es el soporte de interactividad, mediante la cual es posible acceder a un conjunto de aplicaciones y servicios remotos, lo que permite que los usuarios participen de manera activa durante la emisión de un programa de televisión. Ejemplos de aplicaciones interactivas son: encuestas, votaciones, información asociada a un contenido multimedia, consulta del clima, entre otras. Aunque estas se encuentran ampliamente difundidas e implementadas en internet, para desplegarlas y hacerlas usables en el entorno de la televisión es necesario considerar las características y las limitaciones de este medio, dentro de las cuales se encuentran: el tamaño de la letra, los colores que se van a usar, la disposición en pantalla de los contenidos y aplicaciones, las limitaciones para ingresar información desde un control remoto, entre otras. Por lo anterior, es preciso un conjunto de directrices para el diseño de aplicaciones usables, esto con el objetivo de hacer más agradable la experiencia del usuario en televisión.

En el entorno actual, en el que las aplicaciones interactivas están dirigidas a un público cada vez más amplio, a usuarios con menos experiencia en su manejo, la usabilidad es un atributo fundamental para el éxito de dichas aplicaciones. Esta reduce los errores ocasionados por los usuarios y lleva a que realicen las 
tareas de manera más eficiente y efectiva, lo que así aumenta su satisfacción y mejora su experiencia global con la aplicación o sistema con el cual interactúan (Solano, 2012). Con base en lo anterior, este artículo presenta un conjunto de directrices de usabilidad para el diseño de aplicaciones de TDi. Son producto de distintas recomendaciones para el despliegue de contenidos en televisión, así como de evaluaciones de usabilidad realizadas en el Laboratorio Experimental de Televisión Digital de la Universidad del Cauca de Colombia. Se busca que las directrices propuestas las tengan en cuenta diseñadores y desarrolladores en el momento de elaborar este tipo de aplicaciones, de tal manera que tengan mayor aceptación y los usuarios se sientan satisfechos con su uso.

La siguiente sección presenta los referentes teóricos básicos para la comprensión del tema; posteriormente, en la sección 2 se describen los estudios considerados para el desarrollo de este trabajo. La sección 3 presenta los resultados obtenidos en la ejecución de un conjunto de métodos de evaluación de usabilidad. Luego, en la sección 4 se listan las directrices de usabilidad para el diseño de aplicaciones de TDi. Finalmente, se presentan algunas conclusiones y trabajo futuro.

\section{Referentes teóricos}

\subsection{Usabilidad}

El estándar ISO/IEC 9241 define usabilidad como el "grado en el que un producto puede ser utilizado por usuarios específicos para alcanzar objetivos específicos con efectividad, eficiencia y satisfacción en un contexto de uso específico" (ISO, 1998). La efectividad se refiere a la precisión y completitud con que el usuario alcanza objetivos concretos, la eficiencia se refiere a los recursos usados por el usuario para completar estos objetivos y la satisfacción está relacionada con la comodidad y la postura del usuario durante la interacción con el producto.

La evaluación de usabilidad se ha determinado como la actividad que comprende un conjunto de métodos (de inspección y de prueba) que analiza la calidad de uso de un sistema interactivo, en diferentes etapas del ciclo de vida del desarrollo (Nielsen, 1992). Este proceso puede ser llevado a cabo por personas con diferentes habilidades y conocimientos e involucra a usuarios representativos, expertos en usabilidad, entre otros. Es necesario evaluar la usabilidad, para validar que el producto final cumple con los requerimientos y es usable (Granollers, 2007). 


\subsection{Televisión digital interactiva}

La TDi se considera la convergencia de la televisión y las tecnologías de computación, que reúne tres características típicas (Bellotti et al., 2008): interactividad, personalización y digitalización. En la TDi el contenido de un programa, la forma o incluso el orden de presentación puede ser afectado por el usuario. La TDi sobrepasa la televisión analógica en varios aspectos: mejor calidad de imagen y sonido, mejor aprovechamiento del espectro, transmisión de datos simultáneamente, entre otros. Sin embargo, la principal ventaja está en la capacidad de interactuar con el usuario. Esta interactividad le permite ser parte activa de la programación, porque brinda la posibilidad de consultar o extender la información presentada y controlar de cierta manera la secuencia de esta información (Chorianopoulos, 2008).

\subsection{Usabilidad en aplicaciones de televisión digital interactiva}

La facilidad de uso es claramente una prioridad al diseñar aplicaciones interactivas. Gran cantidad de usuarios están acostumbrados a usar un televisor de forma pasiva (prácticamente solo hacer cambios de canales); por ello, al diseñar aplicaciones interactivas claras y lo más intuitivas posibles, se intenta lograr que los usuarios se conviertan en parte activa de un programa de televisión.

Los usuarios están acostumbrados a que la televisión los entretiene y con las innovaciones tecnológicas en las aplicaciones de TDi se incrementa la posibilidad de mejorar el entretenimiento, ya que los usuarios pueden interactuar con los programas que están observando. Por esto es importante centrarse en una apariencia agradable de la aplicación y que esta sea fácil de aprender y entender. Así, otros usuarios pueden ser atraídos a probar las diferentes opciones y características de la aplicación.

La usabilidad va a permitir que los usuarios que utilicen una aplicación de TDi se queden en ella, disfruten de la interacción y quieran volver a ella en el futuro. Para lograr esto, las aplicaciones deben estar diseñadas e implementadas de tal manera que cualquier usuario las utilice y entienda sin problemas.

\section{Trabajos relacionados}

Existen diferentes estudios, como los presentados a continuación, que presentan diferentes iniciativas para apoyar procesos de diseño y evaluación de la usabilidad de aplicaciones en entornos de TDi.

En (Solano et al., 2013), los investigadores proponen un conjunto de heurísticas de usabilidad específicas para aplicaciones de TDi, con el objetivo de ayudar 
en el proceso de evaluación de la usabilidad de este tipo de aplicaciones. Adicionalmente, en este trabajo fue elaborada una lista de verificación (a modo de checklist) para ser usada durante una evaluación heurística. En (Geerts, 2009) y (Rusu et al., 2011) también se plantea un conjunto de heurísticas para televisión digital; los autores de estos trabajos coinciden en que la evaluación heurística es el método de evaluación de usabilidad (MEU) más eficiente y el más utilizado, debido a que es fácil de realizar, económico y permite identificar un buen número de problemas de usabilidad. También en (Hurtado et al., 2012) se plantea un conjunto de directrices de usabilidad relacionadas con la facilidad de aprendizaje y de uso para el diseño de aplicaciones de TDi, las cuales están enfocadas en ciertos perfiles de usuario. Los elementos de estos estudios fueron considerados durante el diseño de las interfaces gráficas, correspondientes a las aplicaciones objeto de estudio (véase sección 3.1).

En (Lee et al., 2008) se afirma que la cantidad y la variedad de directrices es un indicador de lo mucho que falta por explorar en entornos de TDi. Este estudio destaca que no hay suficientes pautas de diseño actualmente disponibles para los diseñadores o evaluadores de este tipo de aplicaciones. Los autores de este trabajo destacan la simplicidad como una de las directrices fundamentales en el diseño de la TDi, puesto que es considerada el factor decisivo de éxito o fracaso para este tipo de aplicaciones. En (Chorianopoulos, 2007) se investiga cómo el concepto genérico de centrado en el usuario se puede traducir en acciones concretas de diseño, desarrollo y evaluación para las interfaces de usuario de la TDi. El conjunto de recomendaciones, herramientas y limitaciones planteadas en estos estudios han permitido extender el concepto de diseño centrado en el usuario para las aplicaciones de TDi.

En (Campo et al., 2010), los investigadores proponen un conjunto de recomendaciones para el despliegue de contenidos de T-Learning. Estas recomendaciones han considerado aspectos técnicos, limitaciones y ventajas de las tecnologías involucradas, aspectos de interacción, navegación en los contenidos y recomendaciones generales, producto del análisis de las tecnologías asociadas al T-Learning, además de la experimentación con aplicaciones y servicios desarrollados en el laboratorio experimental de TDi de la Universidad del Cauca. Este trabajo ha servido como base para identificar posibles directrices de usabilidad en el diseño de aplicaciones de TDi.

Hasta el momento se han desarrollado estudios que exploran diferentes temas relacionados con el diseño de aplicaciones de TDi, los cuales han intentado generar aplicaciones de TDi usables. Dichos estudios proponen métodos, actividades, 
recomendaciones, entre otra información relevante, que se ha tenido en cuenta en el planteamiento de las directrices de usabilidad para el diseño de aplicaciones de TDi.

\section{Evaluación de la usabilidad de aplicaciones de televisión digital interactiva}

Esta sección presenta de forma resumida los resultados obtenidos en la ejecución de un conjunto de MEU en aplicaciones de TDi. Dichos resultados fueron un importante insumo en el momento de proponer las directrices de usabilidad. A continuación se presentan las aplicaciones objeto de estudio para la ejecución de los MEU.

\subsection{Aplicaciones objeto de estudio}

Las aplicaciones objeto de estudio han sido desarrolladas en el Laboratorio Experimental de TDi de la Universidad del Cauca, teniendo en cuenta un conjunto de principios, sugerencias y recomendaciones propuestas en los trabajos relacionados, así como las presentadas en este artículo. Las aplicaciones seleccionadas como objeto de estudio son las siguientes:

- Guía de programación electrónica: ofrece información sobre los programas que van a emitirse en una franja de interés, su descripción, hora de inicio y fin, duración, entre otra. Esta aplicación presenta los programas favoritos y los más vistos o que han obtenido mayores votaciones en encuestas. Los usuarios pueden agregar o quitar programas de la lista de favoritos.

- Tablón o miniblog: permite a los usuarios publicar mensajes o noticias para que sean consultadas o comentadas por otros.

- Chat: permite la comunicación entre grupos de usuarios. El chat funciona en paralelo a un programa de televisión que actúa como foco de la conversación.

Respecto a las aplicaciones objeto de estudio, es importante mencionar que estas se transmiten mediante el estándar tecnológico Digital Video Broadcasting (DVB, 2011), y siguen la especificación Multimedia Home Platform (MHP, 2011). Esto significa que las aplicaciones pueden visualizarse en un televisor (y no en otros dispositivos, como móviles, tabletas, entre otros) mediante el uso de una Set-Top-Box (STB), dispositivo que permite adaptar la señal digital. Adicionalmente, se seleccionaron estas aplicaciones porque en ellas pueden realizarse varias tareas y tienen un buen grado de navegabilidad. 


\subsection{Evaluaciones realizadas}

Con el objetivo de recolectar información sobre la usabilidad de las aplicaciones de TDi objeto de estudio, un conjunto de MEU se llevó a cabo en el Laboratorio Experimental de TDi de la Universidad del Cauca. En cuanto a los métodos de inspección (con expertos), solo fue realizada la evaluación heurística, mientras que por el lado de los métodos de prueba (con usuarios) fueron realizados: experimentos formales, interacción constructiva, entrevistas y cuestionarios. Así, la evaluación heurística, considerada la más eficiente y la más utilizada, sirvió como base de comparación para el análisis de los métodos de prueba de usabilidad.

La evaluación heurística fue hecha por un grupo de cinco evaluadores que inspeccionaron las aplicaciones basándose en un conjunto de heurísticas para aplicaciones de TDi (Solano et al., 2013), lo cual, bajo un análisis sistemático, ayudó a tener una idea sobre el grado de usabilidad de las aplicaciones evaluadas.

Mediante la realización de la evaluación heurística se identificaron un total de 24 problemas de usabilidad por parte de los evaluadores. Estos se agruparon según el principio de usabilidad que incumplen, como se muestra en la tabla 1. Con base en la información de dicha tabla, es evidente la supremacía, en cuanto a cantidad de problemas de usabilidad del principio relación entre el sistema y el mundo real, respecto de los otros principios de usabilidad en los que no se encontraron muchos problemas. Sin embargo, en los principios navegación y flexibilidad y eficiencia de uso la suma de problemas identificados fue significativa. En general, el nivel de criticidad (severidad + frecuencia) de los problemas es un poco alto, un gran número de problemas (16 de 24) fueron calificados, en promedio, con notas mayores a 6 (en una escala de 0 a 8 ). Si bien no son problemas de funcionamiento de las aplicaciones, sí atentan contra la facilidad de uso.

Tabla 1. Preguntas del cuestionario postest

\begin{tabular}{|l|l|l|l|}
\hline N. $^{\circ}$ & \multicolumn{1}{|c|}{ Pregunta } & \multicolumn{1}{|c|}{ Nota menor (1) } & \multicolumn{1}{|c|}{ Nota mayor (5) } \\
\hline 1 & ¿Pudo completar las tareas? & Muy difícilmente & Muy fácilmente \\
\hline 2 & $\begin{array}{l}\text { ¿Considera que la información disponible en } \\
\text { las aplicaciones es completa (suficiente)? }\end{array}$ & Muy en desacuerdo & $\begin{array}{l}\text { Completamente de } \\
\text { acuerdo }\end{array}$ \\
\hline 3 & $\begin{array}{l}\text { La disposición de la información en las } \\
\text { aplicaciones es: }\end{array}$ & Muy difusa & Muy clara \\
\hline 4 & ¿Es fácil la navegación por las aplicaciones? & Muy difícil & Muy fácil \\
\hline 5 & ¿El diseño de las aplicaciones es consistente? & Muy en desacuerdo & $\begin{array}{l}\text { Completamente de } \\
\text { acuerdo }\end{array}$ \\
\hline
\end{tabular}

Continúa 


\begin{tabular}{|l|l|l|l|}
\hline N. $^{\circ}$ & \multicolumn{1}{|c|}{ Pregunta } & \multicolumn{1}{|c|}{ Nota menor (l) } & \multicolumn{1}{|c|}{ Nota mayor (5) } \\
\hline 6 & $\begin{array}{l}\text { Usted califica su grado de satisfacción en el } \\
\text { uso de las aplicaciones. }\end{array}$ & Insatisfactorio & Muy satisfactorio \\
\hline 7 & Volverá a utilizar las aplicaciones. & Muy en desacuerdo & $\begin{array}{l}\text { Completamente de } \\
\text { acuerdo }\end{array}$ \\
\hline 8 & $\begin{array}{l}\text { En comparación con otras aplicaciones } \\
\text { interactivas que usted ha usado, la } \\
\text { experiencia con las aplicaciones de televisión } \\
\text { digital interactiva le ha parecido. }\end{array}$ & Mucho peor & Mucho mejor \\
\hline 9 & $\begin{array}{l}\text { iCómo evalúa su experiencia como } \\
\text { colaborador de esta prueba? }\end{array}$ & Muy desagradable & Muy agradable \\
\hline
\end{tabular}

Fuente: presentación propia de los autores.

Una vez realizada la interpretación de los resultados de la evaluación heurística, los evaluadores identificaron que los problemas con mayor criticidad corresponden principalmente a los principios: relación entre el sistema y el mundo real, flexibilidad y eficiencia de uso, usuarios extraordinarios y control y libertad del usuario. La información recolectada en la evaluación heurística sirvió como base para proponer algunas directrices de usabilidad; sin embargo, los aspectos principales en los cuales están enfocadas las directrices propuestas son: realimentación, consistencia y visibilidad del estado de las tareas/procesos en la aplicación.

Para los métodos de prueba se consideraron dos perfiles de usuario. El primero corresponde a usuarios que tienen un grado de conocimiento aceptable en el uso y desarrollo de aplicaciones de TDi; mientras que el segundo perfil corresponde a usuarios novatos sin conocimiento y experiencia previa en el uso de este tipo de aplicaciones. Los participantes de las pruebas son estudiantes universitarios con una edad entre 20 y 28 años. El análisis de los resultados de cada uno de los MEU realizados sobre las aplicaciones objeto de estudio y los análisis comparativos entre ellos (considerando tiempo invertido en la etapa de planeación, ejecución y análisis de resultados, además de la cantidad y tipo de problemas de usabilidad identificados) permitieron generar el conjunto de directrices para el diseño de aplicaciones de TDi presentadas en la siguiente sección.

La interacción constructiva fue realizada gracias a la participación de 12 usuarios. En este método una pareja de ellos interactúa conjuntamente con la aplicación, mientras verbalizan sus impresiones mutuamente, como en una conversación (Nielsen, 1992). El desarrollo de este método de prueba permitió identificar 25 problemas de usabilidad, entre los cuales se confirmaron los 16 más críticos detectados en la evaluación heurística. Una vez realizada una interacción constructiva, la pareja de usuarios era sometida a una breve entrevista. Las 
entrevistas permitieron encontrar 12 problemas de usabilidad, entre los cuales se confirmaron 5 de los más críticos detectados en la evaluación heurística. Las preguntas realizadas a los usuarios son las siguientes:

1. ¿Es fácil la navegación por la aplicación?

2. ¿La información y las acciones posibles de realizar en la aplicación son fáciles de comprender?

3. ¿Considera que se ofrece la información necesaria para realizar todas las acciones en la aplicación?

4. ¿Siente que tiene el control total sobre la aplicación?

5. ¿Considera que la información presentada a lo largo de la aplicación está organizada de forma adecuada?

6. ¿La aplicación provee una realimentación adecuada a las acciones realizadas?

7. ¿Considera que el diseño de las interfaces es consistente y coherente?

8. ¿Considera que se debe ofrecer a los usuarios una ayuda para especificar cómo usar los elementos de la interfaz?

Los experimentos formales se llevaron a cabo gracias a la participación de 10 usuarios. En este método los usuarios realizan las tareas solicitadas sobre el sistema, mientras los analistas observan la interacción (Nielsen, 1992). Este método permitió identificar 11 problemas de usabilidad, entre los cuales se confirmaron 8 de los más críticos detectados en la evaluación heurística. Los usuarios que participaron en los experimentos eran sometidos a un cuestionario antes (pretest) y después (postest). Mediante el cuestionario pretest se obtuvo información sobre los participantes de la prueba y experiencia en el uso de aplicaciones de TDi; mientras que con el cuestionario postest se obtenía la percepción general de los participantes sobre su experiencia en el uso de las aplicaciones evaluadas. La tabla 1 presenta las preguntas realizadas a los usuarios en el cuestionario postest.

En general, las preguntas de los cuestionarios permitieron obtener resultados alentadores respecto a la satisfacción subjetiva de los usuarios con las aplicaciones de TDi. Hay promedios que superan la nota 4 (en una escala de 1 a 5, donde 5 es la nota máxima correspondiente a una pregunta que se está calificando positivamente), por lo que se puede decir que, en general, los usuarios están conformes con la realimentación, la navegabilidad, la consistencia, el control del usuario, entre otros aspectos. Estos resultados, medianamente satisfactorios, son producto de que durante el diseño y desarrollo de las aplicaciones de TDi 
objeto de estudio fueron considerados diferentes principios, normas y directrices de diseño, incluidas las propuestas en este artículo.

La ejecución de los MEU permitió identificar un conjunto de problemas de usabilidad en las aplicaciones de TDi objeto de estudio, así como proponer un conjunto de soluciones de diseño a partir de dichos problemas. Dicho proceso de evaluación de usabilidad permitió identificar un conjunto de posibles directrices para el diseño de aplicaciones usables en entornos de TDi, las cuales son presentadas en la siguiente sección.

\section{Directrices para diseñar interfaces de aplicaciones de televisión digital interactiva}

Esta sección presenta un conjunto de directrices que deben tenerse en cuenta para el diseño de aplicaciones usables en TDi. Las directrices propuestas se han recopilado a partir de las características de las aplicaciones en TDi (Solano et al., 2011), los resultados de la aplicación de los MEU, la adaptación de las recomendaciones para el despliegue de contenidos en TDi propuestas en (Campo et al., 2010; Lu, 2005) y el trabajo desarrollado en el Laboratorio Experimental de TDi de la Universidad del Cauca. Estas directrices se han clasificado en varias categorías: disposición en pantalla, aplicaciones, navegación y formato. La primera categoría está relacionada con la ubicación del contenido y las aplicaciones interactivas en la pantalla del televisor; la segunda se refiere a las características funcionales de las aplicaciones interactivas; la tercera representa los aspectos para el diseño de la navegación de los contenidos y las aplicaciones interactivas, y la última categoría tiene que ver con el formato que deben tener los contenidos y las aplicaciones interactivas (texto, colores, etc.).

\subsection{Disposición en pantalla}

- De acuerdo con las recomendaciones de despliegue de contenidos en televisión, el contenido multimedia debe ubicarse a la izquierda, y el texto de apoyo, a la derecha, manteniendo una configuración en forma de ele (L) invertida “_l” (figura 1). Por lo anterior, es importante que las aplicaciones no asociadas al contenido estén dispuestas en la parte derecha de la pantalla, ya sea formando una ventana emergente u ocupando un espacio fijo a la derecha del contenido multimedia.

- Para aplicaciones de TDi se sugiere que los títulos o logotipos de programas sean ubicados en la esquina superior izquierda de la pantalla, respetando así 
la distribución en $\mathrm{L}$ invertida y considerando que la lectura en occidente es de izquierda a derecha.

Figura 1. Configuración de la información en forma de L invertida “_|"

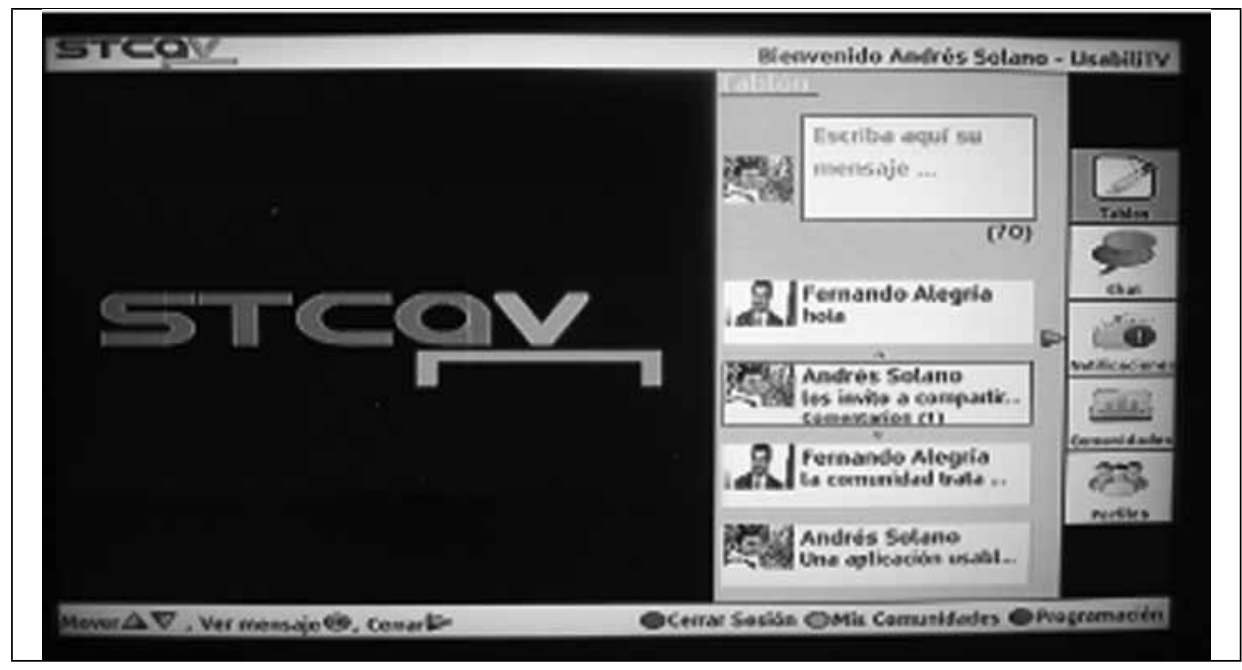

Fuente: presentación propia de los autores.

\subsection{Aplicaciones}

- Un programa de TDi puede contar con dos tipos de aplicaciones: las asociadas o las no asociadas al contenido. Las primeras están ligadas de manera fuerte al contenido multimedia y dependen del contenido que se está visualizando en un determinado momento. Ejemplos de este tipo de aplicaciones son: biografías, votaciones, encuestas, etc. El otro tipo de aplicaciones son independientes del contenido, es decir, están disponibles en cada uno de los programas del entorno de televisión.

- Dado que en televisión el contenido más importante es el multimedia (transmitido por el canal de difusión), las aplicaciones asociadas al contenido deben ser diseñadas como apoyo al contenido central y no para distraer al usuario.

- Considerando que son varias las aplicaciones no asociadas al contenido que pueden ser dispuestas a la derecha del contenido televisivo, se recomienda usar un sistema de pestañas sobre una ventana emergente (figura 1), partiendo así del modelo exitoso de los navegadores de internet. Dicho sistema de pestañas puede quedar anclado en pantalla o como ventana emergente y permitir que el usuario pueda o no ver un programa en la pantalla completa. 
- Para la presentación de contenidos multimedia en conjunto con las aplicaciones no asociadas se sugiere tener la opción de redimensionar la resolución del contenido multimedia cada vez que sea utilizada la ventana emergente de aplicaciones, de tal forma que el contenido televisivo sea el foco principal.

- La interfaz de las aplicaciones de TDi debe ser simple, sin sobrecarga de información, opciones u otros elementos que distraigan al usuario. De acuerdo con la ley de simplicidad de Gestalt (Acevedo et al., 2010), las interfaces de las aplicaciones deben dar impresión de sencillez y ubicar como contenido principal al contenido multimedia.

- La respuesta de los eventos en las aplicaciones interactivas debe ser lo más rápida posible, esto teniendo en cuenta las limitaciones de memoria del STB. Por lo anterior, se recomienda en la etapa de desarrollo hacer uso de protocolos livianos de comunicación y procesamiento de mensajes, como lo son el protocolo de acceso a servicios REST y el formato de mensajes JSON. De igual manera, se recomienda incluir en las aplicaciones no asociadas al contenido, un recomendador de contenidos o aplicaciones, de tal forma que se agilice el proceso de navegación dentro del entorno de televisión.

\subsection{Navegación}

- Dado que en TDi se puede contar en una misma interfaz con aplicaciones asociadas o no al contenido multimedia, es recomendable diseñar la navegación de tal forma que permita distinguir en cuál aplicación se está realizando la interacción.

- El diseño de la navegación para una aplicación de TDi debe ser sencillo y flexible, teniendo en cuenta solo el uso de componentes limitados, como botones numéricos, de colores y flechas. Las flechas se usan para moverse dentro de la interfaz de la aplicación (pestañas o formularios); los botones numéricos, para tomar decisiones o crear atajos entre opciones, y los botones de colores, para desplegar aplicaciones asociadas al contenido o accionar la interacción en los contenidos.

- En cada interfaz se debe mostrar al usuario la posición de navegación (nivel de profundidad de navegación) en la que se encuentra y las opciones de navegación básicas (parte inferior izquierda de la pantalla) (Lu, 2005). De esta forma, el usuario podrá desplazarse e interactuar más fácilmente a través de las opciones de una aplicación.

- Si los botones de colores o de interactividad del control remoto aparecen en la pantalla del televisor, estos deben colocarse de manera horizontal (parte 
inferior izquierda de la pantalla) y en el mismo orden en que aparecen en el control remoto (British Broadcasting Corporation [BBC], 2002); así, la navegación y el control remoto forman parte de un solo sistema de navegación.

- Las aplicaciones de TDi deben proveer opciones de atajos (botones de escape) que permitan cierta flexibilidad a los usuarios experimentados, de tal forma que estos naveguen por las aplicaciones de manera rápida y fácil.

- Con el objetivo de que el usuario tenga control sobre las aplicaciones asociadas o no al contenido, estas deben proporcionar una ruta de escape; así, al pulsar el botón incorrecto, el entorno de televisión debe volver al estado inicial.

- En las interfaces de los contenidos o las aplicaciones interactivas, los botones deben ocupar el mismo lugar en la pantalla, a fin de garantizar coherencia en la navegación (BBC, 2002).

- Cada botón utilizado en una aplicación debe tener una sola etiqueta y función; esto facilita una navegación consistente y un aprendizaje rápido para el usuario.

- Las opciones de navegación deben ser pocas, simples y diferenciar claramente las opciones seleccionadas de las que no lo están. Las aplicaciones de TDi deben ofrecer una secuencia clara de pasos para la realización de tareas. Esto permite que el usuario entienda rápidamente la mecánica de navegación.

- El usuario debe saber siempre dónde está, cómo llegó allí y hacia dónde va. Es necesario que en las interfaces de navegación se presente la ubicación en los títulos, para que los usuarios sepan dónde se encuentran.

- Dadas las restricciones en TDi respecto a tamaño de la letra, poco espacio en la pantalla, ingreso de caracteres desde un control remoto, es necesario usar atajos simples e instrucciones visuales que incrementen la flexibilidad del entorno de TDi.

\subsection{Formato}

- De acuerdo con las recomendaciones para el uso del texto en contenidos de televisión, el tamaño debe ser no menor a 18 puntos en tipo de letra Tiresias. Por eso, tanto las aplicaciones asociadas como las no asociadas al contenido (texto de la ventana emergente) deben cumplir con esta recomendación en el texto empleado.

- Según las recomendaciones de diseño de T-Learning, el color de fondo de los contenidos y aplicaciones en TDi debe mantener contraste: claro-oscuro, respecto al texto; lo anterior para facilitar la lectura a mayor distancia (Chorianopoulos, 2008). 
- En una interfaz de TDi se tiene que utilizar máximo dos tipos de letra, los cuales deben ser distribuidos en títulos, subtítulos, textos de apoyo al contenido multimedia y texto usado en las aplicaciones interactivas. Adicionalmente, el texto usado no debe exceder las 90 palabras por pantalla y las 45 palabras por cuarto de pantalla (BBC, 2002).

\section{Conclusiones y trabajo futuro}

Para que la experiencia de un usuario con una aplicación de TDi sea exitosa y convincente, es necesario desarrollar aplicaciones usables. El conjunto de directrices propuestas busca contribuir en la usabilidad de las aplicaciones de TDi, a fin de garantizar una mejor experiencia interactiva al usuario.

Las directrices de diseño presentadas en este trabajo son un aporte para los diseñadores y actores de la cadena de negocios de TDi, pues sirven como guía para el diseño, la implementación y el despliegue de herramientas y aplicaciones usables en TDi. Mediante la experimentación en el laboratorio de TDi, se encontró que el uso adecuado de estas directrices evita problemas de usabilidad frecuentes.

En las evaluaciones heurísticas es importante que los evaluadores conozcan las características de un sistema usable y tengan cierta experiencia en el dominio de las aplicaciones que se van a evaluar. En este caso de evaluación, los participantes tenían baja/media experiencia evaluando aplicaciones del dominio de la TDi, lo cual pudo influir directamente en la evaluación de usabilidad. Por otro lado, en la realización de los métodos de pruebas se obtuvo una buena realimentación por parte de los usuarios para mejorar gradualmente el diseño de la interfaz de las aplicaciones de TDi evaluadas.

Las directrices para el diseño de aplicaciones usables presentadas en este trabajo se enmarcan en el ámbito de TDi, por lo que podrían ser usadas en diversos campos de aplicación de la televisión, como: T-Learning, T-Commerce, entre otros.

A corto plazo se tienen planeadas otras evaluaciones heurísticas con evaluadores de mayor experiencia en TDi. Adicionalmente, se espera refinar el conjunto de principios heurísticos de acuerdo con lo planteado en Solano et al. (2013) (con las respectivas listas de chequeo), para evaluar la usabilidad de aplicaciones en TDi. También se espera ejecutar otros métodos de prueba con el propósito de evaluar la usabilidad, a fin de identificar cuáles de estos son los más pertinentes para aplicar en entornos de TDi. Finalmente, se pretende generar un conjunto de directrices de usabilidad para otros entornos como TV-móvil y servicio de video bajo demanda (IPTV). 


\section{Referencias}

DVB [web en línea]. 2011. <http://www.dvb.org/> . [Consulta 06-2012].

MHP [web en línea]. 2011. < http://www.mhp.org/>. [Consulta 06-2012].

ACEVEDO, C. P.; ARCINIEGAS, J. L.; GARCÍA, X. y PERRINET, J. Proceso de adaptación de una aplicación de e-aprendizaje a t-aprendizaje. Información Tecnológica. 2010, vol. 21, pp. 27-36.

BELLOTTI, F.; VROCHIDIS, S.; PARISSI, E.; LHOAS, P.; MATHEVON, D.; PELLEGRINO, M.; BO, G. y KOMPATSIARIS, I. A t-learning courses development and presentation framework. IEEE Multidisciplinary Engineering Education Magazine. 2008, vol. 3, pp. 69-76. BRITISH BROADCASTING CORPORATION (BBC). Interactive television style guide: version $2.1,2002$.

CAMPO, W.; CHANCHÍ, G.; URBANO, F. y ARCINIEGAS, J. L. Recomendaciones para el despliegue de contenidos de T-Learning. XI Congreso Internacional Persona-Ordenador, 2010, Valencia, España.

COLLAZOS, C.; RUSU, C.; ARCINIEGAS, J. y RONCAGLIOLO, S. Designing and evaluating interactive television from a usability perspective. Second International Conferences on Advances in Computer-Human Interactions (ACHI 2009), 2009.

COLlaZOS, C. A.; ARCINIEGAS, J.; MONDRAGÓN, V. M. y GARCIA, X. Directrices de diseño y evaluación de la televisión interactiva desde una perspectiva de usabilidad. Interacción 2008. Albacete, España.

CHORIANOPOULOS, K. Re-examination of human-computer interaction methodology in the domain of interactive TV applications [documento en línea]. 2007. < http://www.google.com.co/ $\mathrm{url}$ ? $\mathrm{sa}=\mathrm{t} \& \mathrm{rct}=\mathrm{j} \& \mathrm{q}=\& \mathrm{esrc}=\mathrm{s} \&$ source $=\mathrm{web} \& \mathrm{~cd}=1 \& \mathrm{cad}=\mathrm{rja} \& u a c t=8 \& \mathrm{ved}=0 \mathrm{CCYQ}$ FjAA\&url =http $\% 3$ A\%2F\%2Fwww.researchgate.net $\% 2$ Fpublication $\% 2 F 241090791$ Re-Examination_of_Human-Computer_Interaction_Methodology_in_the_Domain_of_Interactive_TV_Applications\%2Ffile\%2Fe0b4952ce931564af4.pdf $\& \mathrm{ei}=\mathrm{rEcwU} 7 \mathrm{z} 1 \mathrm{MMbz0gG56YGIDw \& usg}=\mathrm{AFQjCNGJKE} 1 \mathrm{D}-3$ Ac-p7c-tEVK1Rnyy1cNA\&bvm=bv.62922401,d.dmQ>.

CHORIANOPOULOS, K. User interface design principles for interactive television applications. International Journal of Human-Computer Interaction. 2008, vol. 24, núm. 6, pp. 556-573.

GEERTS, D. Sociability heuristics for interactive TV supporting the social uses of television. Leuven: Katholieke Universiteit Leuven, 2009.

GRANOLLERS, T. MPI + + a una metodología que integra la ingeniería del software, la interacción persona-ordenador y la accesibilidad en el contexto de equipos de desarrollo multidisciplinares [tesis doctoral]. Lleida: Universidad de Lleida, 2007.

HURTADO, D.; NARVÁEZ, R.; SOLANO, A.; COLLAZOS, C. y ARCINIEGAS, J. L. Directrices de usabilidad relacionadas con la facilidad de aprendizaje y de uso para el diseño de 
aplicaciones de televisión digital interactiva, considerando perfiles de usuario. XII Congreso Internacional de Interacción Persona-Ordenador (Interacción 2012), 2012 Elche, España.

ISO. International Standard ISO/IEC 9241. Ergonomic requirements for office work with visual display terminals, 1998.

LEE, H.; PAUL, F.; CATHAL, G.; ALAN, S.; NOEL, O. C. y HEESEON, P. Balancing the power of multimedia information retrieval and usability in designing interactive TV. International Conference on Designing Interactive User Experiences for TV and Video. ACM, 2008.

LU, K. Interaction design principles for Interactive Television. Georgia: Master of Science in Information Design and Technology, Georgia Institute of Technology, 2005.

NIELSEN, J. The usability engineering life cycle. Computer. 1992, vol. 25, pp. 12-22.

RUSU, C.; RONCAGLIOLO, S.; RUSU, V. y COLLAZOS, C. A methodology to establish usability heuristics. Fourth International Conference on Advances in Computer-Human Interactions (ACHI 2011). 2011, Gosier, Guadeloupe, France.

SOLANO, A. Propuesta metodológica para la evaluación colaborativa de la usabilidad de aplicaciones de televisión digital interactiva [tesis de maestría]. Popayán: Universidad del Cauca, 2012.

SOLANO, A.; CHANCHÍ, G.; COLLAZOS, C.; RUSU, C. y ARCINIEGAS, J. Diseñando interfaces graficas usables de aplicaciones en entornos de televisión digital interactiva. Quinta Conferencia Latinoamericana sobre Interacción Humano-Computador (CLIHC), Porto de Galinhas, Pernambuco, Brasil, 2011.

SOLANO, A.; RUSU, C.; COLLAZOS, C. A. y ARCINIEGAS, J. Evaluando aplicaciones de televisión digital interactiva a través de heurísticas de usabilidad. Ingeniare. Revista Chilena de Ingeniería. 2013, vol. 21, pp. 16-29. 
